\title{
The clinical correlates of barefoot bacteraemia
}

\author{
TJJ Inglis ${ }^{1,2}$
}

Melioidosis is a single infection with a range of syndromic outcomes, ranging from rapidly fatal septicaemia with or without pneumonia, to localised internal organ or soft tissue infection. Five main clinic-pathological disease categories have been described; septicaemia with no, one or multiple foci, and localised focal or multifocal infection without septicaemia (S0, SS, SM, FS, FM, respectively).

In Sri Lanka the common occurrence of lower limb infection with or without septicaemia has been attributed to a barefoot lifestyle, linked to farming, gardening and other activities that put unprotected feet in contact with broken ground. The likely progression from bacterial inoculation through minor cuts and grazes to infection of the lower limbs and eventual bacteraemia presents opportunities for prevention, early diagnosis and improved treatment.

The cutaneous, abscess and joint infection variants of localised melioidosis appear to be underrepresented in the national survey data. Increased recognition coupled with effective treatment may halt progression to septicaemic disease. Awareness of the bacteraemic consequences of a barefoot lifestyle needs to be raised through community health education.

\footnotetext{
${ }^{1}$ School of Pathology and Laboratory Medicine, University of Western Australia, Australia ${ }^{2}$ Department of Microbiology, PathWest Laboratory Medicine. Perth, Western Australia, Australia Address for correspondence: Prof TJJ Inglis, School of Pathology and Laboratory Medicine, University of Western Australia, Australia.+61407994631 Email: tim.inglis@uwa.edu.au ${ }^{10 t t p s: / / o r c i d . o r g / 0000-0002-8559-8245 ~}$
} 